\title{
Validez de las pruebas utilizadas en el diagnóstico inicial y su concordancia con el diagnóstico final en pacientes con sospecha de hiperplasia benigna de próstata
}

\author{
Carballido Rodríguez J*, Badia Llach X**, Gimeno Collado A***, Regadera Sejas L****, \\ Dal-Ré Saavedra R****, Guilera Sardá M.** \\ *Servicio Urología. Hospital Universitario Clinica Puerta de Hierro. Madrid. **Health Outcomes Research \\ Europe. Barcelona. ***Servicio de Urología. Hospital General de la Mancha. Ciudad Real. ****Departamento \\ Médico. GlaxoSmithKline. S.A. \\ Actas Urol Esp. 2006;30(7):667-674
}

\section{RESUMEN}

VALIDEZ DE LAS PRUEBAS UTILIZADAS EN EL DIAGNÓSTICO INICIAL Y SU CONCORDANCIA CON EL DIAGNÓSTICO FINAL EN PACIENTES CON SOSPECHA DE HIPERPLASIA BENIGNA DE PRÓSTATA

Objetivo: Determinar la validez de los métodos diagnósticos al alcance del primer escalón del nivel asistencial [historia clínica (HC), cuestionario I-PSS, tacto rectal (TR) y determinación del antígeno prostático específico (PSA)], para el diagnóstico de la hiperplasia benigna de próstata (HBP).

Población y Métodos: Participaron 363 pacientes atendidos en consultas externas de urología por sospecha de HBP. El diagnóstico inicial de HBP se fue realizando tras la aplicación secuencial de cada una de las siguientes pruebas: 1) HC 2) I-PSS 3) TR y 4) PSA. El diagnóstico emitido tras cada uno de los 4 pasos, se contrastó con el diagnóstico final (gold standard) tras la evaluación de sedimento urinario, volumen miccional residual y tamaño prostático por ecografía y flujometría urinaria. Se realizó un análisis descriptivo y se calculó la validez y concordancia entre cada paso diagnóstico y el gold standard.

Resultados: La sensibilidad, especificidad, valor predictivo positivo y valor predictivo negativo del diagnóstico basado en HC+IPSS+TR+PSA con respecto al diagnóstico final fueron 91\%, 65\%, 95\% y 50\% respectivamente. El porcentaje de acuerdo y el índice kappa entre ambos diagnósticos fueron $87,9 \%$ y 0,5 , respectivamente.

Conclusiones: La concordancia entre el diagnóstico inicial basada en la historia clínica, cuestionario I-PSS, TR y PSA, y el diagnóstico final de HBP es alta, lo que permite recomendar el uso del conjunto de estas pruebas accesibles en el primero de los escalones asistenciales no sólo como primer paso diagnóstico en los pacientes con sospecha de HBP, sino también como estrategia válida para evitar retrasos innecesarios en su manejo inicial y facilitar la adecuada derivación entre los niveles de atención primaria y especializada.

Palabras claves: Hiperplasia benigna de próstata. Diagnóstico. Atención primaria.

\begin{abstract}
VALIDITY OF TESTS FOR INITIAL DIAGNOSIS AND ITS CONCORDANCE WITH FINAL DIAGNOSIS IN PATIENTS WITH SUSPECTED BENIGN PROSTATIC HYPERPLASIA.

Objective: To assess the validity of diagnostic tools available at the primary care setting [medical history (MH), I-PSS questionnaire, digital rectal examination (DRE) and prostate specific antigen (PSA) evaluation] for the diagnosis of benign prostatic hyperplasia (BPH).

Subjects and Methods: 363 patients with suspected prostatic disease referred to urology outpatient clinics were included. For every subject initial BPH diagnosis was collected at each of the following sequential procedures: 1) MH; 2) I-PSS; 3) DRE; and 4) PSA. Each of these diagnostic decisions were compared to a final diagnosis (gold standard) reached after the addition of urinary sediment analysis, ultrasonographic assessment of residual volume and prostatic volume, and peak urinary flow measurement. A descriptive analysis was undertaken; validity and concordance between each diagnostic step and the gold standard was calculated.

Results: Sensitivity, specificity, positive predictive value, and negative predictive value of diagnosis based on $\mathrm{MH}+\mathrm{I}-$ PSS+DRE+PSA with respect to the gold standard were $91 \%, 65 \%, 95 \%$ and $50 \%$, respectively. The percentage of agreement and kappa index between both diagnostic strategies were $87.9 \%$ and 0.5 , respectively.

Conclusions: Concordance between BPH initial diagnosis based on MH+I-PSS+DRE+PSA and diagnosis based on a full range of tests was high, thus allowing recommending the use of this group of initial diagnostic procedures, which are available to the primary care physician, not only as a first diagnostic step in patients with suspected BPH, but also as a valid strategy to prevent unnecessary delay in its initial management and to facilitate appropriate referral from primary to specialized care.
\end{abstract}

Key words: Benign prostatic hyperplasia. Diagnosis. Primary health care. 
$\mathrm{L}^{2}$ a hiperplasia benigna de próstata (HBP), representa el tumor benigno más frecuente en el varón mayor de 50 años y es una patología habitual en la práctica clínica extrahospitalaria. Así, estudios epidemiológicos realizados en España han mostrado una prevalencia global del $11,7 \%$ cuando los criterios clínicos utilizados para su diagnóstico en varones mayores de 40 años contemplaban la presencia de sintomatología miccional moderada-severa, un flujo urinario máximo inferior o igual a $15 \mathrm{ml} / \mathrm{seg}$ y un tamaño prostático de al menos $30 \mathrm{~g}$. Dicha prevalencia se incrementaba hasta el 30\% en pacientes mayores de 70 años ${ }^{1}$.

Diversos estudios realizados hasta la fecha han demostrado que la HBP es una enfermedad de carácter progresivo y con riesgo variable entre unos y otros pacientes. Los factores clínicos predictivos de progresión de la HBP no están todavía definitivamente esclarecidos, pero se han relacionado preferentemente con el volumen prostático y los niveles plasmáticos del antígeno específico prostático (PSA) en el momento del diagnóstico inicial y con el empeoramiento de la de puntuación de sintomas respecto a las cifras basales. Por otro lado, también entre los criterios de progresión se han referido la reducción del flujo urinario máximo y la disminución de la calidad de vida relacionada con los síntomas de $\mathrm{HBP}$ a lo largo del tiempo ${ }^{2,3}$.

El carácter progresivo de la enfermedad y el paulatino envejecimiento de la población, inevitablemente supondrán un incremento de sus tasas de prevalencia así como de los costes sanitarios relacionados con la misma derivados de su morbilidad y repercusión tanto laboral como sociosanitaria ${ }^{4}$. Por esta circunstancia, existe un interés creciente por el desarrollo de estrategias diagnósticas lo más efectivas y eficientes posible.

La evaluación inicial que habitualmente se efectúa en nuestro medio a los pacientes con sintomatología miccional del tramo urinario inferior se fundamenta en la utilización de diferentes pruebas diagnósticas debidamente escalonadas en su indicación y categorizadas de acuerdo con niveles de recomendación y que además sólo deben aplicarse a los pacientes considerados estándar en la evaluación inicial ${ }^{5}$. Esta metodología y criterios de estudio se establecen de acuer- do con las recomendaciones promulgadas por el “Órgano Internacional de Consulta en HBP (Paris 2001)"5. Esta sistemática diagnóstica, en el nivel de recomendación de pruebas imperativas, incluye la realización de una historia clínica detallada de los sintomas urológicos referidos por el paciente, la puntuación de los síntomas miccionales mediante el "International Prostatic Symptom Score” (I-PSS), cuestionario específico reconocido por la Organización Mundial de la Salud $^{6}$ recientemente adaptado y validado en España $^{7}$, el tacto rectal (TR) y la determinación de los niveles plasmáticos del $\mathrm{PSA}^{8}$.

Hasta la actualidad, no nos consta que exista ningún estudio que haya investigado la validez de la combinación de diversas pruebas utilizadas para el diagnóstico de la HBP. De este modo el objetivo del presente estudio fue comparar y establecer el nivel de concordancia entre la metodología diagnóstica utilizada en la evaluación inicial de la HBP basada en la secuencia: historia clínica, cuestionario I-PSS, TR y PSA con respecto a la utilizada al establecer el diagnóstico final realizado al disponer del total de pruebas diagnósticas potencialmente necesarias.

\section{MATERIAL Y MÉTODOS}

Se trata de un estudio prospectivo realizado en consultas externas de urología de 20 centros españoles. Tomaron parte en el estudio un total de 22 urólogos con experiencia mayor o igual a 5 años en esta especialidad con acceso a todas las exploraciones complementarias para el correcto diagnóstico de la HBP y que siguieron en todo momento los criterios asistenciales de la práctica clínica habitual en esta patología. El estudio fue aprobado por el Comité Ético de Investigación Clínica del Hospital Universitario Clínica Puerta de Hierro de Madrid y todos los pacientes tras ser informados firmaron el consentimiento para participar en el mismo.

Los pacientes incluidos en el estudio fueron hombres mayores de 50 años que acudian a las consultas de urología con síntomas miccionales del aparato urinario inferior y a los que no se les había realizado previamente exploraciones complementarias para el diagnóstico de HBP. Se excluyeron todos aquellos pacientes que tuviesen antecedentes de neoplasia prostática, tratamientos 
invasivos previos para la obstrucción del flujo urinario, diabetes mellitus mal controlada y/o neuropatía diabética, antecedentes de alteraciones neurológicas, cirugía pélvica o traumatismo pélvico, enfermedades de transmisión sexual y tratamiento previo con alfa-bloqueantes o inhibidores de la 5-alfa-reductasa.

Los pacientes fueron evaluados en una visita inicial en la que de forma secuencial se realizó una $\mathrm{HC}$ y exploración física, el cuestionario IPSS, el TR, y el valor del PSA. Tras cada uno de estos 4 pasos el médico emitió un juicio diagnóstico provisional sobre la presencia o no de HBP.
Tras conocer el resultado de otras pruebas adicionales (sedimento urinario, volumen residual y tamaño prostático por ecografía abdominal, y flujometría) que se realizaron dentro de las dos semanas siguientes, se emitió el diagnóstico final de HBP (gold standard). El algoritmo diagnóstico del estudio se describe en la Figura 1.

\section{Análisis Estadistico}

Se realizó un análisis descriptivo de las características sociodemográficas y clínicas de la población del estudio. Para cada uno de los pasos del algoritmo diagnóstico se evaluó su validez mediante el cálculo de la sensibilidad (porcentaje de pacientes con sospecha inicial positiva sobre el total de pacientes con diagnóstico final de HBP), la especificidad (porcentaje de pacientes con sospecha inicial negativa entre el total de pacientes sin diagnóstico final de $\mathrm{HBP}$ ), el valor predictivo positivo (VPP) (porcentaje de pacientes con diagnóstico final de HBP con sospecha inicial positiva) y el valor predictivo negativo (VPN) (porcentaje de pacientes sin diagnóstico final de HBP con sospecha inicial negativa), tomando como gold-standard el diagnóstico final de HBP.

La concordancia entre la sospecha diagnóstica inicial después de cada paso en la metodología utilizada y el diagnóstico final se valoró a partir del porcentaje de acuerdo calculado como el número de pacientes con valoraciones coincidentes y el índice Kappa ponderado (IC 95\%).

\section{RESULTADOS}

De la muestra inicial de 363 pacientes, 7 no fueron evaluables, siendo la muestra final para el análisis de 356 pacientes. En la Tabla 1 se muestran 
Tabla 1

Características sociodemográficas

\begin{tabular}{lc}
\hline Variables & $\mathbf{n}=\mathbf{3 5 6}$ \\
\hline Edad (años, media (DE)) & $65,2(8,4)$ \\
Edad de inicio de los síntomas (años, & $62,6(8,2)$ \\
media (DE)) & \\
Tiempo desde inicio síntomas (años, & $2,5(2,6)$ \\
media (DE)) & 63,9 \\
Presencia enfermedades concomitantes (\%) & 22,47 \\
$\quad$ - Hipertensión arterial & 14,6 \\
$\quad$ - Dislipemia & 7,6 \\
$\quad$ - Alergias & 7,0 \\
$\quad$ - Diabetes Mellitus & 7,0 \\
$\quad$ - Cardiopatía isquémica & 5,0 \\
- Enfermedad respiratoria & 52,0 \\
Antecedentes quirúrgicos (\%) & 14,3 \\
\hline Antecedentes familiares de HBP (\%)
\end{tabular}

las características sociodemográficas y clínicas de los pacientes. El rango de edad fue de 50-93 años, y la edad media en el momento del diagnóstico fue de 65,2 años. Un alto porcentaje de pacientes presentaba enfermedades concomitantes $(63,9 \%)$.

\section{Paso 1}

Sintomas según historia clínica y exploración fisica

En la Tabla 2 se muestra la distribución de los sintomas percibidos por el paciente con sospecha de HBP de acuerdo con los datos aportados por la HC. El síntoma comunicado con mayor frecuencia fue la disminución del calibre y fuerza del chorro miccional $(86,7 \%)$, seguido por la frecuencia miccional nocturna que fue mencionada en el $84,3 \%$ de los casos. La distribución de síntomas miccionales obstructivos e irritativos fue similar.

La exploración física puso de manifiesto una palpación abdominal e hipogástrica normal en el $95,5 \%$ y $96,9 \%$ de los casos respectivamente. En el $27,5 \%$ de ellos se detectó

\section{Tabla 2}

alguna alteración en la exploración de los genitales externos (fimosis, hernia o hidrocele).

\section{Paso 2}

Síntomas según la puntuación de la escala IPSS La puntuación media (DE, rango) del I-PSS fue de 15,4 puntos $(5,6,3-35)$. En la Tabla 2 se presentan los síntomas ordenados por orden de frecuencia, siendo nuevamente la disminución del calibre y fuerza del chorro $(97,4 \%)$ y la frecuencia miccional nocturna los sintomas mencionados con mayor frecuencia. La distribución de los síntomas de acuerdo con su carácter obstructivo o irritativo fue también similar.

En la Tabla 3 se muestra la distribución de pacientes según la puntuación en el cuestionario I-PSS (preguntas $n^{\circ}$ 1-7) y la pregunta sobre calidad de vida (pregunta $\mathrm{n}^{\mathrm{o}}$ 8). La mayoría de los pacientes mostraron síntomas moderados $(67,4 \%)$ y estar insatisfechos con su calidad de vida relacionada con la sintomatología miccional $(45,2 \%)$.

\section{Pasos 3 y 4}

Tacto rectal y PSA

El TR describió la glándula como fibroelástica (94\%), lisa (92,7\%), de movilidad normal (96,6\%), con límites definidos (93\%) y no dolorosa $(85,9 \%)$. Con respecto al tamaño estimado mediante esta exploración la glándula fue pequeña, mediana o grande, en el 36,9\%, 49,3\% y 13,8\% de los casos, respectivamente. El porcentaje de acuerdo con el tamaño prostático observado por ecografía fue del $64,5 \%$.

Sintomatología percibida según Historia Clínica inicial e I-PSS

\begin{tabular}{lclc}
\hline $\begin{array}{l}\text { SínTOMAS PROSTÁTICOS } \\
\text { Historia Clínica }\end{array}$ & $\%$ & I-PSS* & $\%$ \\
\hline $\begin{array}{l}\text { Disminución del calibre y } \\
\text { fuerza del chorro }\end{array}$ & 86,7 & $\begin{array}{l}\text { Disminución del calibre y fuerza } \\
\text { del chorro }\end{array}$ & 97,4 \\
Frecuencia miccional nocturna & 84,3 & Frecuencia miccional nocturna & 95,4 \\
Goteo posmiccional & 62,3 & Polaquiuria & 90,8 \\
Polaquiuria & 61,4 & Tenesmo & 87,9 \\
Micción intermitente & 48,7 & Micción intermitente & 86,1 \\
Sensación de micción incompleta & 42.9 & Urgencia & 83.8 \\
Urgencia-Incontinencia & 25,2 & Prensa abdominal & 82,9 \\
Otros & 20,3 & & \\
\hline
\end{tabular}

*I-PSS: pacientes con presencia de síntomas: respuesta entre 1-5. 
Tabla 3

Distribución de síntomas y calidad de vida según el cuestionario I-PSS.

\begin{tabular}{lclc}
\hline $\begin{array}{l}\text { SíNTOMAS según I-PSS } \\
\text { (preguntas } \mathrm{n}^{\circ} \text { 1 - 7) }\end{array}$ & $\%$ & $\begin{array}{l}\text { Calidad de Vida } \\
\text { (pregunta } \mathrm{n}^{\circ} \text { 8) }\end{array}$ & $\%$ \\
\hline Leve (0-7) & 7,6 & Satisfechos (0-2) & 28,2 \\
Moderado (8-19) & 67,4 & Indiferentes (3) & 26,5 \\
Severo (20-35) & 24,9 & Insatisfechos (4-6) & 45,2 \\
\hline
\end{tabular}

El valor medio (DE) de PSA fue de $2,7 \mathrm{ng} / \mathrm{mL}$ $(3,8)$ con valores mínimo y máximo de entre 0 y $64 \mathrm{ng} / \mathrm{mL}$, respectivamente. El 75,6\% de los pacientes presentaron valores de PSA entre 0-4 $\mathrm{ng} / \mathrm{mL}$; ligeramente elevado $(4,1-9,9 \mathrm{ng} / \mathrm{mL})$ en el $22,9 \%$ y valores moderado-altos $(\geq 10 \mathrm{ng} / \mathrm{mL})$ en el $1,4 \%$ de los pacientes.

\section{Paso 5}

Sedimento, ecografia abdominal y flujometría

La analítica de orina proporcionó datos sospechosos de infección urinaria en el 6.8\% de los pacientes. La ecografía abdominal mostró un volumen residual medio (DE) de 58,7 (71) mL y el volumen prostático medio (DE) fue de 46 (43) mL. En la flujometría se observó una media (DE) de flujo máximo de $12(4,7) \mathrm{mL} / \mathrm{seg}$ para un volumen miccional total medio (DE) de 226, $1(94,7) \mathrm{mL}$.

\section{Validez y concordancia diagnósticas}

En la Tabla 4 se muestra el porcentaje de diagnósticos de HBP en cada paso del algoritmo, que aumentó de forma progresiva en cada paso. El diagnóstico final (gold standard) demostró que 313 (87,9\%) de los 356 pacientes evaluados tenían HBP.

\section{Tabla 4}

Resultados de las pruebas diagnósticas de HBP

\begin{tabular}{llc}
\hline Prueba Diagnóstica & \multicolumn{2}{c}{ RESULTADo (n, \%) } \\
Positivo & Negativo \\
\hline 1. Historia clínica & $279(78,4)$ & $77(21,6)$ \\
2. I-PSS & $280(78,6)$ & $76(21,4)$ \\
3. Tacto Rectal & $283(79,5)$ & $73(20,5)$ \\
4. PSA & $299(84,2)$ & $57(16,0)$ \\
5. Diagnóstico final & $313(87,9)$ & $43(12,1)$ \\
\hline
\end{tabular}

Diagnóstico final: basado en la HC, I-PSS, TR, PSA, sedimento urinario, ecografia y flujometría.
En la Tabla 5 se muestran los resultados de validez y concordancia. La sensibilidad, especificidad, VPP y VPN aumentaron también en cada paso diagnóstico, siendo de $91 \%, 65 \%, 95 \%$ y $50 \%$, respectivamente, en el paso 4 $(\mathrm{HC}+\mathrm{I}-\mathrm{PSS}+\mathrm{TR}+\mathrm{PSA}) \cdot \mathrm{El}$ porcentaje de pacientes clasificados correctamente respecto al gold standard en cada paso y el índice kappa aumentaron también al ir añadiendo pruebas diagnósticas, siendo máximos en el paso 4, 87,9\% y 0,5, respectivamente. El 71,6\% (255/356) de los pacientes mantuvo el mismo diagnóstico desde el primer paso (HC).

\section{DISCUSIÓN}

La HBP es una de las enfermedades más frecuentes entre los varones a partir de los 50 años y aumenta progresivamente con la edad ${ }^{9}$. Si consideramos el envejecimiento progresivo de la población española en los próximos años, se estima un incremento importante de esta patología $^{10}$. La importancia del diagnóstico precoz tiene como objetivo primordial su correcta identificación y tratamiento inicial para intentar modificar la progresión de la enfermedad y las complicaciones derivadas de la misma, entre ellas el desarrollo de retención urinaria aguda y la necesidad de tratamiento quirúrgico. En una mayoría de países está aumentando el papel que juega la asistencia primaria en el manejo terapéutico de esta enfermedad, escalón asistencial al que se considera determinante $^{11}$. Es importante por tanto, estudiar y resaltar el valor de la sospecha inicial de HBP basada en los síntomas, el TR y la determinación del PSA, herramientas fundamentales en la orientación diagnóstica y terapéutica desde los primeros pasos en la asistencia sanitaria a estos pacientes; asímismo es relevante conocer el grado de concordancia entre el diagnóstico inicial hecho con estas herramientas del primer escalón asistencial y el diagnóstico final de HBP que se alcanza con todo el arsenal diagnóstico especializado. 
Tabla 5

Validez y concordancia entre la sospecha diagnóstica inicial y el diagnóstico final de HBP.

\begin{tabular}{lcccc}
\hline $\begin{array}{l}\text { Validez } \\
\text { Paso Diagnóstico }\end{array}$ & $\begin{array}{c}\text { Sensibilidad } \\
\text { (\% (IC 95\%)) }\end{array}$ & $\begin{array}{c}\text { Especificidad } \\
\text { (\% (IC 95\%)) }\end{array}$ & $\begin{array}{c}\text { VPP } \\
\text { (\%) }\end{array}$ & $\begin{array}{c}\text { VPN } \\
\text { (\%) }\end{array}$ \\
\hline 1: HC & $80,2(75,8-84,6)$ & $34,9(20,6-49,1)$ & 90,0 & 19,5 \\
2: HC+IPSS & $82,1(77,8-86,3)$ & $46,5(31,6-61,4)$ & 91,8 & 26,3 \\
3: HC+IPSS+TR & $85,3(81,4-89,2)$ & $62,8(48,3-77,2)$ & 94,3 & 37,0 \\
4: HC+IPSS+TR+PSA & $91,0(87,8-94,2)$ & $65,1(50,9-79,4)$ & 95,0 & 50,0 \\
\hline
\end{tabular}

VPP = Valor Predictivo Positivo; VPN = Valor Predictivo Negativo.

\section{Concordancia \\ Paso Diagnóstico}

1: $\mathrm{HC}$

2: $\mathrm{HC}+\mathrm{IPSS}$

3: $\mathrm{HC}+\mathrm{IPSS}+\mathrm{TR}$

4: $\mathrm{HC}+$ IPSS+TR+PSA
Porcentaje de acuerdo

(\%)

74,7

77,7

82,6

87,9
Índice Kappa (IC 95\%)

$0,11(0-0,22)$

$0,21(0,10-0,33)$

$0,37(0,25-0,49)$

$0,50(0,37-0,63)$

mayor impacto en su calidad de vida relacionada con su salud y función sexual ${ }^{16}$. La repercusión sobre la calidad de vida del paciente relacionada con la presencia de sintomatología miccional atribuible a HBP se evaluó con una única pregunta que se incluye en el cuestionario I-PSS. Después de agrupar las respuestas por categorías, casi la mitad de los pacientes refirió algún grado de insatisfacción con sus sintomas. De todos modos, nuevamente podemos presuponer que la procedencia de los pacientes del presente estudio explica el

Los síntomas comunicados con mayor frecuencia tras la HC y el I-PSS fueron la disminución del calibre y fuerza del chorro y la frecuencia miccional nocturna. Diversos estudios han evaluado el tipo y frecuencia de los sintomas asociados con la HBP. Un estudio realizado en España entre varones mayores de 50 años de la población general que aplicó el I-PSS, encontró el incremento de la frecuencia miccional nocturna $(59,7 \%)$, la polaquiuria $(43,3 \%)$ y la disminución en la fuerza y calibre del chorro $(36,5 \%)$ como los síntomas reportados con mayor frecuencia ${ }^{12}$. Las diferencias entre estos datos y los del presente estudio pueden deberse a las diferencias entre las poblaciones estudiadas, pacientes que consultan por la presencia de sintomas en el presente estudio, frente a una muestra de la población general. Del mismo modo, en este estudio la mayoría de pacientes mostraban sintomas moderados o severos, siendo este porcentaje más bajo en otros estudios realizados en España en la población general $(30,4 \%)^{13}$. Datos de distintos países del mundo como Escocia, Francia, EE.UU. y Japón, han confirmado que los sintomas más frecuentes en la población general son polaquiuria, frecuencia miccional nocturna, urgencia y goteo postmiccional $2,14,15$.

A pesar de ser los síntomas obstructivos los que se comunican con mayor frecuencia en la mayoría de estudios, son los irritativos los que preocupan más al enfermo prostático, debido al que puedan presentar situaciones clinicas más evolucionadas o con peor tolerancia a las mismas. Estudios que han evaluado la calidad de vida relacionada con problemas urológicos en la población general, han encontrado porcentajes inferiores de pacientes insatisfechos ${ }^{12}$.

La concordancia entre el diagnóstico de HBP basado en los síntomas percibidos por los pacientes al realizar la HC y el I-PSS, y el diagnóstico final tras aplicar todas las exploraciones complementarias fue alta, $74,7 \%$ y $77,7 \%$ respectivamente, hecho que confirma la validez del cuestionario I-PSS ${ }^{6}$. En el global de la muestra, un $71,6 \%$ de los pacientes mantuvieron el mismo diagnóstico durante todo el estudio. La combinación de la HC, I-PSS, TR y PSA, fue la estrategia diagnóstica que mostró mayor sensibilidad y VPP, aunque, por otro lado, hay que mencionar la menor especificidad y VPN observados. Estos resultados se justifican por tratarse de pacientes atendidos en consultas de urología en los que la prevalencia de la HBP es alta. El porcentaje de concordancia con el diagnóstico final e índice de kappa, 87,9\% y 0,5 respectivamente, definen el acuerdo entre diagnósticos como bueno ${ }^{17}$.

En el momento actual, los algoritmos recomendados para el diagnóstico de la HBP, con independencia de su ámbito de utilización en la práctica clínica, incluyen en el primer paso la anamnesis y administración del cuestionario I$\mathrm{PSS}^{18}$. Por otro lado, se ha confirmado que los 
resultados del I-PSS son equivalentes cuando el cuestionario es auto-administrado o cuando es el médico quien lo administra, lo cual facilita su utilización y difusión ${ }^{7,19}$. Los resultados de este estudio confirman que la mejor estrategia para un diagnóstico inicial de HBP, comprende, además de la identificación de los síntomas evidenciados en la anamnesis y tras la administración del IPSS, la realización de un TR y la determinación del PSA.

La mayoría de pacientes mostraron una próstata de tamaño mediano en la exploración rectal y dentro de los límites esperables en la HBP. Del mismo modo, la mayor parte de pacientes mostraron valores de PSA inferiores a $4 \mathrm{ng} / \mathrm{mL}^{20}$. No obstante, en el $24 \%$ de los pacientes de esta serie el valor basal del PSA estaba por encima de los rangos considerados en este estudio como normales $(<4 \mathrm{ng} / \mathrm{mL})$. Esta circunstancia, sin duda debe interpretarse en el contexto de la evidencia actual que correlaciona el volumen prostático en la HBP y los valores del PSA no sólo en su evaluación basal sino también como factor de respuesta terapéutica a los fármacos inhibidores de la enzima 5-alfa reductasa tanto en monoterapia como en terapia de combinación ${ }^{21,22}$. En este sentido, es oportuno destacar la trascendencia práctica de este hallazgo en el contexto del diagnóstico precoz del cáncer de próstata y la controversia recientemente planteada respecto a la interpretación de los valores normales del PSA cuando se introducen variables como la edad y el volumen glandular prostático ${ }^{23}$.

Consideramos que la procedencia de los pacientes, consultas externas de urología, no limitan la relevancia de los resultados obtenidos en el estudio ni impiden su extrapolación a otros escalones asistenciales, ya que la secuencia establecida en la utilización de las pruebas diagnósticas se fijó intentando reproducir el orden que parece lógico seguir ante un paciente nuevo que consulte por síntomas de sospecha de HBP y responde a la categorización de las pruebas diagnósticas establecidas por el Órgano Internacional de Consulta de la HBP (OMS 2001) ${ }^{5}$.

Los resultados obtenidos en este estudio confirman la importancia del uso de exploraciones básicas, a las que se tiene acceso en cualquier consulta del primer escalón asistencial para el diagnóstico de la HBP y en consecuencia no sólo no retrasa el diagnóstico de la enfermedad sino que también contribuye a optimizar los criterios de derivación entre distintos escalones asistenciales.

En resumen, entre pacientes con sospecha de HBP atendidos en consultas de urología, los síntomas más frecuentes se distribuyen por igual entre los de carácter irritativo y obstructivo, refiriendo síntomas moderados una amplia mayoría de los pacientes y mostrándose insatisfechos con su calidad de vida relacionada con esta sintomatología casi la mitad de los mismos. Nuestro estudio confirma el valor de los síntomas clínicos, el TR y el valor del PSA, en el diagnóstico de la HBP $\mathrm{y}$ por tanto se puede recomendar el uso de este conjunto de pruebas como primer paso diagnóstico en los pacientes con sospecha de HBP posibilitando además el diseño de nuevas estrategias asistenciales en estos pacientes. Asímismo, estos datos justifican la realización de estudios en escenarios asistenciales específicos como los de atención primaria en nuestro medio para poder corroborar estos hallazgos.

\section{Investigadores y centros participantes en el estudio}

Javier Azpárren, Hospital Donosti, San Sebastián

Isidre Bonet, Hospital de la Creu Roja, Barcelona

Carlos Dondériz, Centro de especialidades Torrentes, Valencia

Maximino Lozano, Clínica San Francisco, León

Fructuoso García, Clínica San Francisco, León

Ignacio Mora, Clínica Girona, Girona

Félix Millán, Clínica Puigvert, Barcelona

Gilberto Chechile, Clínica del Remei, Barcelona

Jesús Guajardo, Hospital Arnau de Vilanova, Lleida

Francisco Ramírez, Hospital Carlos Haya, Málaga

Alberto Budia, Hospital La Fe, Valencia

Joaquín Carballido, Hospital Puerta de Hierro, Madrid

Paloma Cárcamo, Hospital La Paz, Madrid

Argimiro Collado, Hospital Elche, Alicante

Alfonso Gimeno, Hospital Gral. de la Mancha, Alcázar de San Juan

Miguel Hevia, Hospital General de Asturias, Oviedo

Andrés López de Alda, Hospital Don Benito, Badajoz

Ignacio Martínez-Sapiña, Hospital Meixoeiro, Vigo

Rafael Medina, Hospital Virgen del Rocío, Sevilla

Juan Pedro San Millán, Hospital La Paz, Madrid

Ernesto Sánchez, Hospital Virgen de la Macarena, Sevilla

Francisco Sánchez, Hospital Verge de la Cinta, Tortosa 


\section{REFERENCIAS}

1. Chicharro-Molero JA, Burgos-Rodriguez R, Sanchez-Cruz JJ, Rosal-Samaniego JM, Rodero-Carcia P, RodriguezVallejo JM. Prevalence of benign prostatic hyperplasia in Spanish men 40 years old or older. J Urol. 1998;159(3): 878-882.

2. Chute CG, Panser LA, Girman CJ, Oesterling JE, Guess HA, Jacobsen SJ, et al. The prevalence of prostatism: a population-based survey of urinary symptoms. J Urol. 1993;150(1): 85-89.

3. Anderson JB, Roehrborn CG, Schalken JA, Emberton M. The progresión of benign prostatic hyperplasia: examining the evidence and determining the risk. Eur Urol. 2001; 39:390-399.

4. Madersbacher S, Alivizatos G, Nordling J, Rioja C, Emberton M, de la Rosette J. EAU 2004 Guidelines on Assessment, therapy and follow-up of men with Lower Urinary Tract Symptons suggestive of benign prostatic obstruction (BPH Guidelines). Eur Urol. 2004;46:547-554.

5. Chatelain L, Denis L, Foo JK. Evaluation and treatment of lower urinary tract symptoms in older man. In: Recommendations of the International Scientific Committee. Health Publication Ltd. 2001;8:519-534.

6. Barry MJ, Fowler FJ, Jr., O’Leary MP, Bruskewitz RC, Holtgrewe HL, Mebust WK et al. The American Urological Association symptom index for benign prostatic hyperplasia. The Measurement Committee of the American Urological Association. J Urol. 1992;148(5):1549-1557.

7. Badia X, Garcia-Losa M, Dal-Re R, Carballido J, Serra M. Validation of a harmonized Spanish version of the IPSS: evidence of equivalence with the original American scale. International Prostate Symptom Score. Urology 1998;52(4): 614-620.

8. Unda Urzaiz M., Rodriguez Alcantara F, Badia X., Garcia Losa M, Carballido Rodriguez J., Dal-Re Saavedra R. Características sociosanitarias y diagnósticas del paciente prostático en España a finales del siglo XX. Actas Urol Esp 2001;25(3):200-206.

9. Berry SJ, Coffey DS, Walsh PC, Ewing LL. The development of human benign prostatic hyperplasia with age. J Urol. 1984;132(3):474-479.

10. Carballido Rodriguez JA, Rodriguez Vallejo JM, Llano Senaris JE. Hiperplasia prostática benigna y medicina basada en la evidencia: su aproximación a la práctica clínica. Med Clin. (Barc) 2000;114 Suppl 2:96-104.

11. Chapple CR. Lower urinary tract symptoms suggestive of benign prostatic obstruction-Triumph: design and implementation. Eur Urol 2001; 39 Suppl 3:31-36.

12. Gomez Acebo A., Rodriguez Vallejo JM., Rodriguez Mora V, Garcia Alcazar I. Calidad de vida y sintomatología en la hiperplasia prostática benigna en la población activa española. Med Clin (Barc) 2000;114 Suppl 3:81-89.
13. Hunter DJ, Berra-Unamuno A, Martin-Gordo A. Prevalence of urinary symptoms and other urological conditions in Spanish men 50 years old or older. J Urol. 1996;155(6): 1965-1970.

14. Sagnier PP, MacFarlane G, Richard F, Botto H, Teillac P, Boyle P. Results of an epidemiological survey using a modified American Urological Association symptom index for benign prostatic hyperplasia in France. J Urol. 1994;151(5): 1266-1270.

15. Garraway WM, Collins GN, Lee RJ. High prevalence of benign prostatic hypertrophy in the community. Lancet 1991;338:469-471.

16. Scarpa RM. Lower urinary tract symptoms: what are the implications for the patients? Eur Urol.. 2001;40 Suppl 4: 12-20.

17. Landis JR, Koch GG. The measurement of observer agreement for categorical data. Biometrics 1977;33(1):159-174.

18. Roehrborn CG, The potential of serum prostate-specific antigen as a predictor of clinical response in patients with lower urinary tract symptoms and benign prostatic hyperplasia. BJU Int. 2004;93 Suppl 1:21-26.

19. Plante M, Corcos J, Gregoire I, Belanger MF, Brock G, Rossingol M. The international prostate symptom score: physician versus self-administration in the quantification of symptomatology. Urology 1996;47(3):326-328.

20. Carroll P, Coley C, McLeod D, Schellhammer P, Sweat G, Wasson $\mathrm{J}$ et al. Prostate-specific antigen best practice policy-part I: early detection and diagnosis of prostate cancer. Urology 2001;57(2):217-224.

21. Roehrborn CG, Boyle P,Gould AL, Waldstreiher J. Serum prostate-specific antigen as a predictor of prostate volume in men with benign prostatic hyperplasia. Urology 1999;53(3):581-589.

22. McConnell JD, Roehborn CG, Bautista OM, Andriole GL Jr, Dixon CM, Kusek JW, et al. The long term effect of doxazosin, finasteride and combination therapy on the clinical progression of benign prostatic hyperplasia. N Eng $\mathrm{J}$ Med. 2003;349:2387-2398.

23. Stamey TA, Caldwell M, McNeal JE, Nolley R, Hemenez M, Downs J. The Prostate Specific Antigen era in the United States is over for prostate cancer: What happened in the Last 20 years? J Urol. 2004;172(4 Pt1):1297-1301.

\author{
Dr. X. Badia \\ Health Outcomes Research Europe \\ Avda. Diagonal 618, 1 C-D \\ Barcelona 08021 \\ E-mail: xbadia@hor-europe.com
}

(Trabajo recibido el 5 de abril de 2006) 